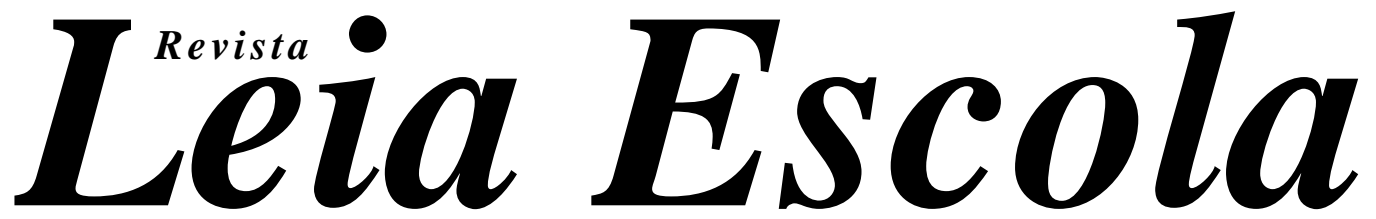

Programa de Pós-Graduação em Linguagem e Ensino 
UNIVERSIDADE FEDERAL DE CAMPINA GRANDE

Reitor Vicemário Simões

Vice-reitor Camilo Allyson Simões de Farias

Diretora do Centro de Humanidades Fernanda de Lourdes Almeida Leal

UNIDADE ACADÊMICA DE LETRAS

Coordenadora Administrativa Márcia Candeia Rodrigues

Coordenadores de Graduação

Licenciatura em Letras Espanhol

Licenciatura em Letras Francês

Licenciatura em Letras Inglês

Licenciatura em Letras Libras

Licenciatura em Letras Português

Coordenadora do Programa de Pós-Graduação em

Linguagem e Ensino

Coordenadora de Pesquisa e Extensão

Isis Milreu

Carmen Verônica A. R. Nóbrega

Cleydstone Chaves dos Santos

Aline Risseli Florindo Silva

Edmilson Luiz Rafael

Manassés Morais Xavier

Denise Lino de Araújo

Neide de Fátima Cesar da Cruz

Editora da Universidade Federal de Campina Grande Diretor Administrativo

Revista Leia Escola

Editora Chefe

Comissão Editorial

Quadriênio 2018-2021

José Hélder Pinheiro Alves

ISSN 2358-5870

Márcia Tavares

Denise Lino de Araújo

Maria Augusta G. de Macedo Reinaldo

Naelza de Araújo Wanderley

Corpo Editorial Aldinida Medeiros (UEPB)

André Pinheiro (UFPI)

Angela Paiva Dionisio (UFPE)

Amarino Queiroz (UFRN)

Ana Cristina Marinho Lúcio (UFPB)

Ana Virgínia Lima (UFRN)

Bruno Alves Pereira (UEPB)

Darcília Simões (UERJ)

Daniela Segabinazi (UFPB)

Derivaldo dos Santos (UFRN)

Edenize Ponzo Peres (UFES)

Edmilson Luiz Rafael (UFCG)

Eliana Kefalas Oliveira (UFAL)

Fabiana Oliveira (UFAL)

Fabiele Stockmans de Nardi (UFPE)

Fabiano Tadeu Grazioli (URI/FAE)

Fábio Marques de Souza (UEPB)

Fernanda Aquino Sylvestre (UFU)

Givaldo Melo de Santana (UFSE)

Josilene Pinheiro Mariz (UFCG)

José Hélder Pinheiro Alves (UFCG)

José Veranildo Lopes da Costa Junior (UERN)

Karina Chianca (UFPB)

Lílian de Oliveira Rodrigues (UERN)

Luiz Francisco Dias (UFMG)

Maria Amélia Dalvi (UFES)

Marco Antônio Margarido Costa (UFCG)

Maria Angélica de Oliveira (UFCG)

Maria Auxiliadora Bezerra (UFCG)

Maria Hozanete Alves de Lima (UFRN)

Maria Marta dos Santos Silva Nóbrega (UFCG)

Marie Hélène Torres (UFSC)

Renata Junqueira de Souza (UNESP)

Roberto Carlos de Assis (UFPB)

Sílvia Pilegi Rodrigues (UFMT)

Simone Dália de Gusmão Aranha (UEPB)

Sinara de Oliveira Branco (UFCG)

Valdenides Cabral Dias (UFRN)

Os trabalhos publicados são de responsabilidade exclusiva dos seus autores $\quad$ ISSN 2358-5870 Capa: Leila Leite Santana - UERN

Leia Escola - Revista do Programa de Pós-Graduação em Linguagem e Ensino da UFCG.

v. 20, n. 2, 2020. Campina Grande: EDUFCG, 2020.

1. Linguística

2. Linguística Aplicada

3. Literatura

4. Ensino 
UNIVERSIDADE FEDERAL DE CAMPINA GRANDE CENTRO DE HUMANIDADES

UNIDADE ACADÊMICA DE LETRAS
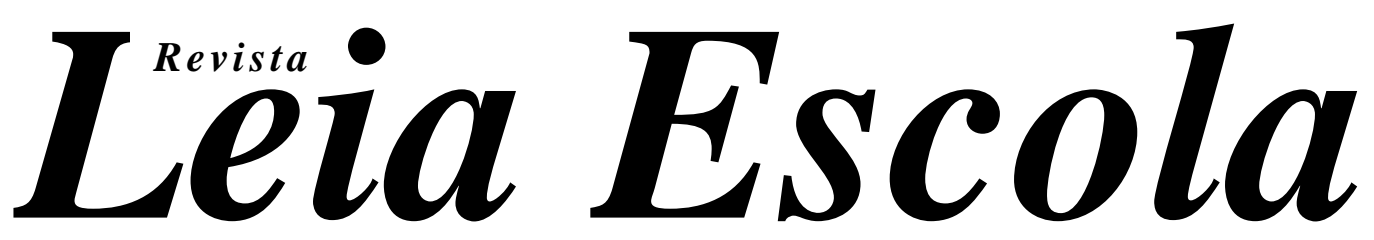

Programa de Pós-Graduação em Linguagem e Ensino

\title{
ENSINO E APRENDIZAGEM DE LÍNGUAS E LITERATURAS NOS INSTITUTOS FEDERAIS
}

\author{
Organizadores \\ Prof. Dr. Antonio Ferreira da Silva Júnior \\ Universidade Federal do Rio de Janeiro \\ Prof. Dr. José Veranildo Lopes da Costa Junior \\ Universidade do Estado do Rio Grande do Norte \\ Prof. Dr. Marcel Alvaro de Amorim \\ Instituto Federal do Rio de Janeiro \\ Universidade Federal do Rio de Janeiro
}

\section{EDUFCG}

Campina Grande

2020 


\section{SUMÁRIO}

\section{DOSSIÊ TEMÁTICO}

O TEU SOTAQUE É BONITO OU FEIO? UM ESTUDO SOBRE CRENÇAS E ATITUDES LINGUÍSTICAS DE ALUNOS DO ENSINO MÉDIO DO IFMS DE NOVA ANDRADINA/MS

Márcio Palácios de Carvalho e Patrícia Gacriela da Rocha.

ONLINE COLLABORATIVE WRITING IN ENGLISH AT THE FEDERAL INSTITUTE OF RIO GRANDE DO NORTE: SOME QUANTITATIVE DATA ON STUDENT PARTICIPATION IN INTEGRATED SECONDARY EDUCATION

Sabrina Guedes Miranda Dantas e Samuel de Carvalho Lima.

NAS TRAMAS DO NHANDUTI: TECENDO A IGUALDADE DE GÊNERO NUM INSTITUTO FEDERAL DE FRONTEIRA

Eli Gomes Castanho e Fabrícia Carla Viviani.

PRODUÇÃO TEXTUAL NO ENSINO DE LÍNGUA ESPANHOLA: UMA EXPERIÊNCIA COM O LIVRO CARTONERO NO CEFET/RJ

Fabiana Oliveira de Souza...

ESTÁGIO SUPERVISIONADO NO CURSO DE LICENCIATURA DE LETRAS NO IFES: DESAFIOS, DEBATES E PROPOSTAS NA FORMAÇÃO INICIAL

Selma Lúcia de Assis Pereira.

O QUE PODE ESTA LÍNGUA? CONSIDERAÇÕES SOBRE O ENSINO DE PORTUGUÊS EM CURSOS SUPERIORES DO IFSP

Valmir Luis Saldanha da Silva

REPRESENTAÇÕES SOCIAIS DE ALUNOS ACERCA DA REDAÇÃO DO ENEM: PRÁTICAS DE ENSINO DE ESCRITA DO INSTITUTO FEDERAL DO PARANÁ

Cláudia Cândido da Silva e Carmen Teresinha Baumgärtner.

ENSINO CRÍTICO DE LÍNGUA PORTUGUESA NO IFSP - CAMPUS PRESIDENTE EPITÁCIO

Irando Alves Martins Neto

O PROJETO DE CURSO EM DEBATE A PARTIR DE FALAS DE PROFESSORES DA LICENCIATURA EM ESPANHOL DO INSTITUTO FEDERAL DE BRASÍLIA

EXPERIÊNCIAS SIGNIFICATIVAS DE LEITURA E ESCRITA DO TEXTO LITERÁRIO NO IFPB

Sayonara Abrantes de Oliveira Uchoa, Symara Abrantes Albuquerque de Oliveira Cabral, Henrique Miguel de Lima Silva. 
AS QUESTÕES ÉTNICO-RACIAIS NAS AULAS DE INGLÊS INSTRUMENTAL DO IFRJ SOB A ÓTICA DA PEDAGOGIA DOS MULTILETRAMENTOS

Vanessa Moreno Mota e Heloise Vasconcellos Gomes Thompson.

LEITURA E INTERPRETAÇÃO DE TEXTO NO IFPR CAMPUS JACAREZINHO: LETRAMENTO CRÍTICO PARA A CIDADANIA

David José de Andrade Silva.....

A REPRESENTATIVIDADE DOS INSTITUTOS FEDERAIS NO VII CONGRESSO LATINO-AMERICANO DE FORMAÇÃO DE PROFESSORES DE LÍNGUAS

Aline Moraes de Carvalho e Mônica Bomtempo Reis Soares

TRABALHO DIDÁTICO COM TEXTO LITERÁRIO E VISIBILIDADE AFROLATINA NAS AULAS DEEAE: REFLEXÕES SOBRE NOSSA EXPERIÊNCIA NO IFRJ

Paula Fernanda Vicente Rosa.

PRÁTICAS DE LEITURA E DE ESCRITA NO IFG: DA PONTA DO LÁPIS AOS MULTILETRAMENTOS

Dalva Ramos de Resende Matos, Pauliana Duarte Oliveira e Selma Zago da Silva Borges

A FORMAÇÃO DO LEITOR EM SUAS MÚLTIPLAS DIMENSÕES: A LEITURA LITERÁRIÁ SEDIMENTANDO A PRÁTICA INTEGRADORA NO ENSINO MÉDIO TÉCNICO NO IFPB - CAMPUS JOÃO PESSOA

Girlene Marques Formiga, Maria da Conceição Monteiro Cavalcanti e Clebianne Vieira de Araújo

LEITURA LITERÁRIA NO ENSINO MÉDIO DO INSTITUTO FEDERAL CATARINENSE, CAMPUS SÃO BENTO DO SUL

Raquel Cardoso de Faria e Custódio e Ana Paula Pereira Villela.

\section{ARTIGOS}

EL ESPAÑOL EN LA EDUCACIÓN BRASILEÑA: DESDE EL HISTÓRICO NACIONAL HASTA LAS ESPECIFICIDADES EN LAS IES DE RIO GRANDE DO NORTE

Tatiana Lourenço de Carvalho,José Veranildo Lopes da Costa Junior.

LEITURA E PRODUÇÃO DE SENTIDOS EM CHARGES MEDIADAS PELO WHATSAPP NO ENSINO FUNDAMENTAL

Edivânia Helena Nunes, Benedito Gomes Bezerra e Maria Ladjane dos Santos Pereira........

LEITURA DOS ESTEREÓTIPOS DA MULHER NEGRA BRASILEIRA EM "VIAJE AL OTRO BRASIL"

Ruan Fellipe Munhoz

PROFISSIONALIDADE DOCENTE PARA O TRABALHO PEDAGÓGICO EM AMBIENTES DIGITAIS NO ENSINO E APRENDIZAGEM DE LÍNGUAS

Manoela Oliveira de Souza Santana. 
EDUCAÇÃO ESCOLAR FUNDADA EM NOVOS MODOS DE ESCRITA: ALFABETIZAÇÃO SEM ESCRITA CURSIVA

Sinval Martins de Sousa Filho, Pedro Henrique da Silva...

\section{ENTREVISTA}

PROGRAMA BALE: ONDE LER É RESISTIR; É PARTILHAR SONHOS; É TRANSFORMAR VIDAS! - Entrevista com Maria Lúcia Pessoa Sampaio 